(at 1-minute interval) during surgery, were collected. Using the preoperative noninvasive and intraoperative invasive systolic blood pressure values, we calculated the average real variability (ARV), known as a reliable representation of time series blood pressure variability. Associations between the ARV index and survival outcomes were investigated.

Results: In total, 441 patients were included. Using the integer close to the median as a cut-off value for the ARV, we found that the high-ARV group $(\geq 8 ; n=220)$ showed worse progression-free survival (PFS) than the low-ARV group $(<8$; $\mathrm{n}=221$ ) (median $=82.8 \%$ vs. $89.6 \%$; $=0.020$ ). In multivariate analysis adjusting for confounders, ARV $\geq 8$ was identified as an independent poor prognostic factor for PFS (adjusted hazard ratio $[\mathrm{HR}]=1.887$; 95\% confidence interval $[\mathrm{CI}]=1.158-3.076$; $\mathrm{p}=0.011)$. In the subgroup of open RH $(\mathrm{n}=238), A R V \geq 8$ was associated with significantly worse PFS (adjusted HR=2.402; 95\% CI=1.119-5.155; $\mathrm{p}=0.024)$. In contrast, in the subgroup of MIS RH ( $n=238)$, PFS did not differ by the ARV index.

Conclusion: The ARV index, indicating intraoperative hemodynamic instability, might be a novel prognostic biomarker for disease recurrence in early cervical cancer patients who receive primary open $\mathrm{RH}$, not MIS RH.

Oral (OC3)

Cervical Cancer

https://doi.org/10.3802/jgo.2021.32.S1.0C3

\section{Comparison of locally advanced cervical cancer treatment guidelines in Asia}

\author{
David Tan, ${ }^{1, *}$ Bradley J. Monk, ${ }^{2}$ Jitender Takyar, ${ }^{3}$ \\ José David Hernández Chagüi, ${ }^{4}$ Takayuki Enomoto, ${ }^{5}$ Eric \\ Pujade-Lauraine $^{6}$ \\ 'National University Cancer Institute, Singapore \\ (david_sp_tan@nuhs.edu.sg) \\ ${ }^{2}$ University of Arizona College of Medicine, Tucson, AZ, USA \\ ${ }^{3}$ Parexel International, Mohali, India \\ ${ }^{4}$ AstraZeneca, Gaithersburg, MD, USA \\ ${ }^{5}$ Niigata University Graduate School of Medical and Dental Sciences, Niigata,
} Japan 6ARCAGY-GINECO, Paris, France

Objective: A previous global systematic literature review (SLR) assessing recommended treatment for locally advanced cervical cancer (LACC) revealed international consensus on the use of concurrent chemoradiotherapy (CCRT) as standard of care (SoC) for stage IIB-IVA LACC. However, recommendations for stage IB2-IIA LACC varied. We present a subanalysis of Asian guidelines to determine if recommended LACC treatment is consistent across Asia.

Methods: The most recent English-language cervical cancer treatment guidelines and consensus statements were identified from literature databases (1999-2020), national authority websites, and bibliographies. For comparison, additional, nonEnglish, Asian country guidelines were translated. Results: Eleven guidelines from 5 Asian countries were reviewed; 10 were last updated between 2013-2021, 1 was undated. Seven guidelines provided treatment recommendations by disease stage using International Federation of Gynecology and Obstetrics (FIGO) 2009 staging criteria. For stage IB2-IIA2 disease, surgery, cCRT, or RT alone was recommended. Cisplatin-based cCRT followed by brachytherapy was SoC for suitable patients with stage IIBIVA LACC among all Asian guidelines, except Japan, where radical hysterectomy or cCRT was recommended for stage IIB. For stage IVA disease, 2 Indian guidelines (ICMR and NCGI) discussed the use of palliative treatments versus curative intent therapy. Non-SLR guidelines from Vietnam and Malaysia were consistent with this consensus.

Conclusion: In line with global SLR findings, consensus on cCRT as primary treatment for stage IIB-IVA LACC was recommended by Asian guidelines. For stage IB-IIA LACC, recommendations varied between radical hysterectomy and cCRT, or cCRT/RT alone. Alignment of guidelines with FIGO 2018 staging criteria may reduce variation in recommended treatment for early-stage LACC.

Funding: AstraZeneca.

Oral (OC4)

Cervical Cancer

https://doi.org/10.3802/jgo.2021.32.S1.0C4

\section{Adjuvant pelvic radiation versus observation in intermediate-risk early-stage cervical cancer patients following radical surgery: a propensity score analysis}

Thunwipa Tuscharoenporn, Tanarat Muangmool, Kittipat Charoenkwan*

Chiang Mai University, Chiang Mai, Thailand (kittipat.c@cmu.ac.th)

Objective: To compare survival outcomes, posttreatment complications, and quality of life of early-stage cervical cancer patients classified as intermediate-risk group following primary radical surgery between the patients who received adjuvant pelvic radiation and those without adjuvant treatment. Methods: Two hundred and thirty stage IB-IIA cervical cancer patients who had radical hysterectomy and pelvic lymphadenectomy from January 2003 to December 2018 and were classified as having intermediate-risk according to the Sedlis's criteria were included. The participants were divided 